\title{
Seguimiento continuo del proceso de gelificación de alcóxidos de silicio y titanio mediante ensayos reológicos
}

\author{
E. RUDÉ, J. LLORENS y C. MANS \\ Departament d’Enginyeria Química i Metal·lúrgia. Universitat de Barcelona. 08028 Barcelona
}

\begin{abstract}
En este trabajo se ha estudiado el proceso de gelificación desde un nuevo punto de vista: el seguimiento de la transición solgel mediante la evolución con el tiempo de la distribución de tiempos de relajación, que está directamente relacionada con la distribución de pesos moleculares. La técnica utilizada para la determinación de la distribución de tiempos de relajación es la reología. El proceso de gelificación se ha seguido de forma continua mediante dos ensayos reológicos distintos: (1) ensayos dinámicos u oscilatorios y (2) ensayos de retardo y recuperación, con el objetivo de caracterizar de forma completa la viscoelasticidad del sistema a lo largo de todo el proceso. El estudio ha abarcado todos los estadios de la gelificación: (a) el estadio pre-gel, (b) el propio estadio gel y (c) el estadio post-gel. Los procesos estudiados corresponden a soles poliméricos de titanio y de silicio. Los resultados obtenidos permiten evaluar la influencia de la composición de los soles, así como comparar los dos tipos de soles analizados. Además, permiten describir el tiempo de gelificación desde una nueva perspectiva, basada en la distribución de tiempos de relajación, ya que se observa que este momento corresponde a una distribución de polidispersidad máxima.
\end{abstract}

Palabras clave: proceso sol-gel, soles poliméricos de titanio y silicio, propiedades viscoelásticas, espectros de tiempos de relajación, tiempo de gelificación

\section{Continuous monitoring of the gelation process of silicon and titanium alkoxides through rheological measurements}

In the present work the gelation process has been studied from a new point of view: monitoring the sol-to-gel transition through the evolution of the distribution of relaxation times, which is directly related to the molecular weight distribution. The relaxation times distribution has been determined from rheological measurements. The gelation process has been continuously monitored by two different rheological tests: (1) dynamic or oscillatory tests and (2) creep and recovery tests, to characterise the viscoelasticity of the system throughout the whole process in a complete way. The study includes all the states of the gelation process: (a) pre-gel state, (b) gel state and (c) post-gel state. The analysed processes have been the polymerisation of titanium and silica sols. The influence of the composition of the sols has been discussed and the two different types of sols have been compared. Furthermore, the gel point is described from a new viewpoint based on the relaxation spectrum: at the gel point, the polidispersity of the relaxation times distribution reaches a maximum.

Key words: sol-gel process, titanium and silica polymeric sols, viscoelastic properties, relaxation times spectra, gel time

\section{INTRODUCCIÓN}

Actualmente hay un gran interés científico en el desarrollo de la técnica de la reología como herramienta para determinar la distribución de pesos moleculares (DPM) de un sistema polimérico y, por tanto, caracterizarlo estructuralmente. De hecho, en los últimos tiempos, se ha constatado como una tecnología adecuada y precisa para la determinación de distribuciones de pesos moleculares de sistemas poliméricos lineales, ya sean monodispersos o polidispersos (1-7).

Sin embargo, la investigación realizada hasta el momento actual en la obtención de información estructural a partir de información reológica en sistemas que están reaccionando, y en particular en sistemas que están gelificando, es prácticamente nula. La mayoría de trabajos que existen en la literatura centran su atención en la caracterización de la transición sol-gel únicamente desde el punto de vista reológico, adqui- riendo últimamente la reología dinámica (dentro de la viscoelasticidad lineal) un papel destacado como medida directa del cambio de comportamiento líquido a sólido que tiene lugar en la transición sol-gel (8-13).

En el trabajo que se presenta a continuación se ha querido dar un paso más y obtener información estructural a partir de dicha caracterización viscoelástica del proceso de gelificación. Para ello, se ha analizado el proceso mediante la evolución de la distribución de tiempos de relajación (DTR), que relaciona la función viscoelástica espectro de relajación, $\mathrm{H}(\lambda)$, con el tiempo de relajación. Es sabido que el tiempo de relajación de una molécula, $\lambda_{\mathrm{i}}$, está directamente relacionado con su peso molecular, $\mathrm{M}_{\mathrm{i}}$, siguiendo una relación de tipo potencial, así como la distribución de tiempos de relajación lo está con la distribución de pesos moleculares del sistema $(14,15)$. 


\section{PROCEDIMIENTO EXPERIMENTAL}

\subsection{Materiales y métodos de preparación de los soles}

\subsubsection{SOLES DE SILICIO}

Los soles poliméricos de silicio estudiados se han preparado con diferentes proporciones de los siguientes componentes: tetraetoxisilano (TEOS) $\left[\mathrm{Si}\left(\mathrm{OC}_{2} \mathrm{H}_{5}\right)_{4}\right]$, etanol absoluto $\left[\mathrm{C}_{2} \mathrm{H}_{5} \mathrm{OH}\right]$, ácido clorhídrico $37 \%[\mathrm{HCl}]$ y agua desionizada purificada con un sistema Millipore $\left[\mathrm{H}_{2} \mathrm{O}\right]$. Los parámetros composicionales independientes que gobiernan las reacciones de hidrólisis y policondensación que tienen lugar durante el proceso de gelificación son: la concentración de alcohol, $\mathrm{A}=[\mathrm{EtOH}](\% \mathrm{~mol})$, la razón molar de agua, $\mathrm{r}=\left[\mathrm{H}_{2} \mathrm{O}\right] /[\mathrm{Si}]$ y la razón molar de catalizador, $\mathrm{P}=[\mathrm{HCl}] /[\mathrm{Si}]$. A los diferentes soles estudiados se les ha asignado un nombre de acuerdo con su composición, tal como se muestra en la Tabla I.

En la literatura se describen dos métodos para la preparación de soles de silicio: el procedimiento de hidrólisis en una única etapa $(16,17)$ y el de hidrólisis en dos etapas $(18)$. Dichos métodos, tal como su nombre indica, se diferencian por la forma de adicionar el agua: en un solo paso o en dos. El método de hidrólisis en una etapa consiste en mezclar primero el alcohol con el alcóxido y añadir a continuación el agua con el catalizador, agitando posteriormente la mezcla de reacción durante un período de tiempo determinado. El método de hidrólisis en dos etapas, en cambio, consiste en mezclar inicialmente parte del agua necesaria con el alcohol y el alcóxido, y al cabo de un cierto tiempo de agitación añadir la parte restante de agua junto con el catalizador.

En el presente trabajo se ha utilizado el primero de los procedimientos descritos, aunque ligeramente modificado para evitar posibles problemas de inmiscilibidad entre el TEOS y el $\mathrm{H}_{2} \mathrm{O}$. Esta modificación consiste en añadir el alcohol total necesario en dos etapas. Así pues, se preparan dos soluciones por separado: por un lado, se añade parte del alcohol sobre el alcóxido y se mezcla enérgicamente durante $30 \mathrm{~s}$; por otro lado, se prepara la solución hidrolizante, mezclando el alcohol restante y las cantidades correspondientes de $\mathrm{H}_{2} \mathrm{O}$ y $\mathrm{HCl}$. A continuación se colocan ambas soluciones en un baño a $50^{\circ} \mathrm{C}$ (temperatura de trabajo), donde se mantienen durante aproximadamente $1 \mathrm{~h}$. Por último, se añade la solución hidrolizante sobre la solución que contiene el alcóxido y se mezcla enérgicamente durante $1 \mathrm{~min}$, teniendo lugar una reacción exotérmica que da como resultado un sol incoloro y transparente, sobre el cual se realizará el estudio reológico.

\subsubsection{SOLES DE TITANIO}

Para la preparación de los soles poliméricos de titanio, los componentes utilizados en distintas proporciones son los siguientes: tetraisopropilortotitanato (TIPOT) $\left.\left[\mathrm{Ti}^{(\mathrm{OPr}}\right)_{4}\right]$, ácido acético glacial $\left[\mathrm{CH}_{3} \mathrm{COOH}\right]$, isopropanol $\left[\mathrm{CH}_{3} \mathrm{CHOHCH}_{3}\right]$, ácido nítrico $70 \%\left[\mathrm{HNO}_{3}\right]$ y agua desionizada purificada con un sistema Millipore $\left[\mathrm{H}_{2} \mathrm{O}\right]$. Los parámetros composicionales independientes que en este caso gobiernan las reacciones de hidrólisis y policondensación que tienen lugar, son cuatro: la concentración molar final de precursor, $\mathrm{C}=[\mathrm{Ti}]$, la razón molar de sustitución, $\mathrm{S}=[\mathrm{AcOH}] /[\mathrm{Ti}]$, la razón molar de agua, $\mathrm{r}=\left[\mathrm{H}_{2} \mathrm{O}\right] /[\mathrm{Ti}]$ y la razón molar de catalizador, $\mathrm{P}=\left[\mathrm{HNO}_{3}\right] /[\mathrm{Ti}]$. Análogamente a los soles de silicio, se ha asignado un nombre a los distintos soles estudiados de acuerdo con su composición (ver Tabla II).
TABLA I. NOMENCLATURA Y RAZONES MOLARES CORRESPONDIENTES A LOS SOLES DE SILICIO ESTUDIADOS.

\begin{tabular}{lccc}
\hline Muestra & $\mathbf{A}$ & $\mathbf{r}$ & $\mathbf{P}$ \\
\hline [Si] A30r4.00P0.100* & 30 & 4,00 & 0,100 \\
[Si] $\underline{\mathbf{A} 50 \mathbf{r} 4.00 \mathbf{P} 0.100}$ & 50 & 4,00 & 0,100 \\
[Si] A30 $\mathbf{r} 9.00 \mathbf{P} 0.100$ & 30 & 9,00 & 0,100 \\
[Si] A30r4.00P0.050 & 30 & 4,00 & 0,050 \\
\hline
\end{tabular}

* Sol estándar

TABLA II. NOMENCLATURA Y RAZONES MOLARES CORRESPONDIENTES A LOS SOLES DE TITANIO ESTUDIADOS $(\mathrm{C}=0.58 \mathrm{~mol} \cdot \mathrm{dm}-3)$.

\begin{tabular}{|c|c|c|c|}
\hline Muestra & $\mathbf{S}$ & $\mathbf{r}$ & $\mathbf{P}$ \\
\hline$[\mathrm{Ti}] \underline{\mathrm{S} 1.50} \mathbf{r} 3.27 \mathrm{P} 0.110$ & 1,50 & 3,27 & 0,110 \\
\hline [Ti] S2.10r3.27P0.110* & 2,10 & 3,27 & 0,110 \\
\hline [Ti] S2.10 $\underline{\mathbf{r} 3.50 \mathbf{P} 0.110}$ & 2,10 & 3,50 & 0,110 \\
\hline 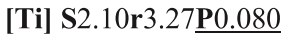 & 2,10 & 3,27 & 0,080 \\
\hline
\end{tabular}

* Sol estándar

La preparación de los soles de titanio estudiados se lleva a cabo mediante un procedimiento en dos etapas: la modificación química del precursor alcóxido con ácido acético, seguida de la hidrólisis del alcóxido modificado. El procedimiento utilizado se describe con detalle en una publicación anterior (20).

\subsection{Ensayos reológicos}

Las medidas reológicas se han realizado de forma continua con un reómetro de control del esfuerzo (HAAKE RS100) y un sensor cono-placa con un diámetro de $60 \mathrm{~mm}$ y un ángulo de $4^{\circ}\left(\mathrm{C} 60 / 4^{\circ}\right)$. El sensor ha sido aislado debidamente del exterior mediante un dispositivo especial, de diseño propio, para evitar problemas de evaporación del solvente. La temperatura de trabajo ha sido de $50^{\circ} \mathrm{C}$ y $30^{\circ} \mathrm{C}$ para los soles de silicio y titanio, respectivamente.

Se han llevado a cabo dos tipos de ensayos reológicos: ensayos dinámicos $\mathrm{u}$ oscilatorios y ensayos de retardo y recuperación. Los ensayos oscilatorios consisten en imponer a la muestra un esfuerzo sinusoidal, $\sigma$, con una amplitud máxima $\sigma_{0} \mathrm{y}$ una frecuencia $\omega$ :

$$
\sigma=\sigma_{0} \sin \omega \mathrm{t}
$$

y medir la deformación resultante, $\gamma$, que también varía de forma sinusoidal con una amplitud máxima $\gamma_{0} \mathrm{y}$ con la misma frecuencia $\omega$, pero desfasada un ángulo $\delta$ :

$$
\gamma=-\gamma_{0} \sin (\omega t+\delta)
$$

Esta deformación resultante puede descomponerse en dos partes (19):

$$
\begin{aligned}
\gamma & =-\sigma_{0}(A \cos \delta \sin \omega t+A \sin \delta \cos \omega t)= \\
& =-\sigma_{0}\left(G^{\prime} \sin \omega t+\eta^{\prime} \omega \cos \omega t\right)
\end{aligned}
$$


siendo A la relación entre las amplitudes máximas: $A=\gamma_{0} / \sigma_{0}$

La componente de la deformación resultante en fase con el esfuerzo aplicado define el módulo elástico o de almacenamiento, $\mathrm{G}^{\prime}\left[\mathrm{G}^{\prime}=\mathrm{A} \cos \delta\right]$, mientras que la componente en desfase $90^{\circ}$ define la viscosidad dinámica, $\eta^{\prime}\left[\eta^{\prime}=\mathrm{A} \sin \delta / \omega\right]$, ambos función de la frecuencia aplicada. El módulo elástico es sensible a la deformabilidad de la estructura del sistema y la viscosidad dinámica está relacionada con la energía viscosa que se disipa durante la aplicación del esfuerzo. Así pues, un ensayo oscilatorio proporciona, a una determinada frecuencia, simultáneamente dos propiedades del sistema: elasticidad y viscosidad.

Las distintas estructuras que componen el sistema tienen diferentes tiempos de relajación. Se entiende por tiempo de relajación de una estructura el tiempo transcurrido desde que cesa la perturbación a la cual está siendo sometida la estructura hasta que alcanza una nueva situación de equilibrio. El tiempo de relajación de una estructura está en relación con su tamaño: a mayor tamaño mayor tiempo de relajación. Las estructuras manifiestan elasticidad cuando se someten a esfuerzos que oscilan a frecuencias cuyos inversos son menores que sus tiempos de relajación y manifiestan viscosidad cuando los inversos de esas frecuencias son mayores que sus tiempos de relajación. Por tanto, una estructura manifiesta viscosidad y elasticidad simultáneamente cuando se somete a esfuerzos que oscilan a una frecuencia cuyo inverso es del orden de su tiempo de relajación.

Por lo que se refiere a los ensayos de retardo y recuperación, éstos consisten en aplicar un esfuerzo constante, $\sigma_{0}$ durante un período de tiempo, $t, t_{0} \leq t<t_{1}$, que se retira cuando $t=t_{1}$ y medir la evolución de la deformación resultante con el tiempo. La función viscoelástica que caracteriza el material en este tipo de ensayo es la capacitancia, $\mathrm{J}(\mathrm{t})$, que se define como el cociente entre la deformación y el esfuerzo aplicado:

$$
J(t)=\frac{y(t)}{\sigma_{0}}
$$

Es conocido que

los materiales viscoelásticos presentan un comportamiento cuadrático a tiempos pequeños y lineal a tiempos elevados, siguiendo en dicha zona la siguiente ecuación (19):

$$
\mathrm{J}(\mathrm{t})=\mathrm{J}_{\mathrm{e}}^{0}+\frac{\mathrm{t}}{\eta_{0}}
$$

donde $\mathrm{J}_{\mathrm{e}}^{0}$ es la capacitancia

de flujo estacionario y una medida de la energía elástica almacenada en el material durante el ensayo y $\eta_{0}$ la viscosidad límite a gradiente cero y una medida de la viscosidad del sistema. Así pues, nuevamente se obtienen las dos propiedades del sistema: elasticidad y viscosidad. Sin embargo, en este caso se trata de la elasticidad y viscosidad a frecuencia cero, ya que corresponde al comportamiento del material cuando el tiempo de ensayo es elevado, frecuencia que, por otro lado, no puede explorarse con un ensayo dinámico. Por tanto, la información obtenida con los ensayos de retardo y recuperación complementa la información obtenida a partir de los ensayos dinámicos. Por este motivo, en este trabajo se han realizado los dos tipos de ensayos para caracterizar de forma completa el comportamiento viscoelástico de todas las estructuras del material.

Dado que durante la transición sol-gel el sistema reacciona y evoluciona de forma continua desde una solución de precursor molecular de baja viscosidad a un sólido viscoelástico de viscosidad infinita, las condiciones experimentales de análisis no pueden mantenerse constantes a lo largo del seguimiento del proceso de gelificación. De hecho deben modificarse para conseguir precisión en los resultados, así como garantizar que se trabaja bajo unas condiciones tales que no modifican la estructura macromolecular del material (zona de viscoelasticidad lineal). Por este motivo se han realizado una serie de ensayos previos para determinar el esfuerzo máximo que soporta el material sin que se altere su estructura en los distintos estadios de la gelificación.

\section{RESULTADOS Y DISCUSIÓN}

En este apartado se analizará la evolución con el tiempo del módulo de almacenamiento, $\mathrm{G}^{\prime}(\omega)$, la viscosidad dinámica, $\eta^{\prime}(\omega)$, el módulo plateau, $G_{N^{\prime}}^{0}$ la viscosidad límite a gradiente cero, $\eta_{0}$ y la capacitancia de flujo estacionario, $\mathrm{J}_{\mathrm{e}}^{0}$. Todas y cada una de estas funciones y parámetros viscoelásticos son necesarios para el cálculo posterior de las distribuciones de tiempos de relajación.

$\mathrm{Al}$ analizar los resultados se observa que las funciones y propiedades viscoelásticas de todos los sistemas estudiados, independientemente de la naturaleza y composición del sol, siguen la pauta general de evolución propia de un sistema que está gelificando. Inicialmente la viscosidad y elasticidad del sistema son muy bajas y prácticamente constantes, aumentando de forma brusca en un tiempo de reacción característico, conocido como tiempo de gelificación, $\mathrm{t}_{\mathrm{g}}$ momento a partir del cual continúan aumentando pero ya de forma mucho más suave.

Si se analiza para cada tipo de sol (en base silicio o en base titanio) la influencia que ejerce la composición sobre la viscosidad y la elasticidad del sistema, se observa que todos los sistemas, a pesar de tener tiempos de gelificación muy diferentes (ver Tablas III y IV), rinden comportamientos viscoelásticos significativamente iguales. El tipo de sol, sin embargo, sí que proporciona comportamientos distintos. De ahí que los resultados se presenten en una gráfica tipo para cada una de las funciones y/o parámetros viscoelásticos y tipo de sol.

TABLA III. TIEMPOS DE GELIFICACIÓN CORRESPONDIENTES A LOS SOLES DE

\begin{tabular}{|c|c|}
\hline Muestra & $\mathbf{t}_{\mathrm{g}}[\mathrm{h}]$ \\
\hline [Si] A30r4.00P0.100* & 8,47 \\
\hline$[\mathbf{S i}] \underline{\mathbf{A} 50} \mathbf{r} 4.00 \mathbf{P} 0.100$ & 53,7 \\
\hline [Si] A30 $\underline{\mathbf{r} 9.00 \mathbf{P} 0.100}$ & 25,9 \\
\hline$[\mathbf{S i}] \mathbf{A} 30 \mathbf{r} 4.00 \underline{\mathbf{P} 0.050}$ & 23,0 \\
\hline
\end{tabular}
SILICIO ESTUDIADOS.

* Sol estándar

TABLA IV. TIEMPOS DE GELIFCACIÓN CORRESPONDIENTES A LOS SOLES DE TITANIO ESTUDIADOS.

\begin{tabular}{lc}
\hline Muestra & $\mathbf{t}_{\mathrm{g}}[\mathbf{h}]$ \\
\hline [Ti] $\underline{\mathbf{S} 1.50 \mathbf{r} 3.27 \mathbf{P} 0.110}$ & 0,77 \\
[Ti] S2.10r3.27P0.110* & 2,7 \\
[Ti] S2.10 $\underline{\mathbf{r} 3.50 \mathbf{P} 0.110}$ & 1,2 \\
[Ti] S2.10r3.27吕0.080 & 2,0 \\
\hline
\end{tabular}

* Sol estándar 


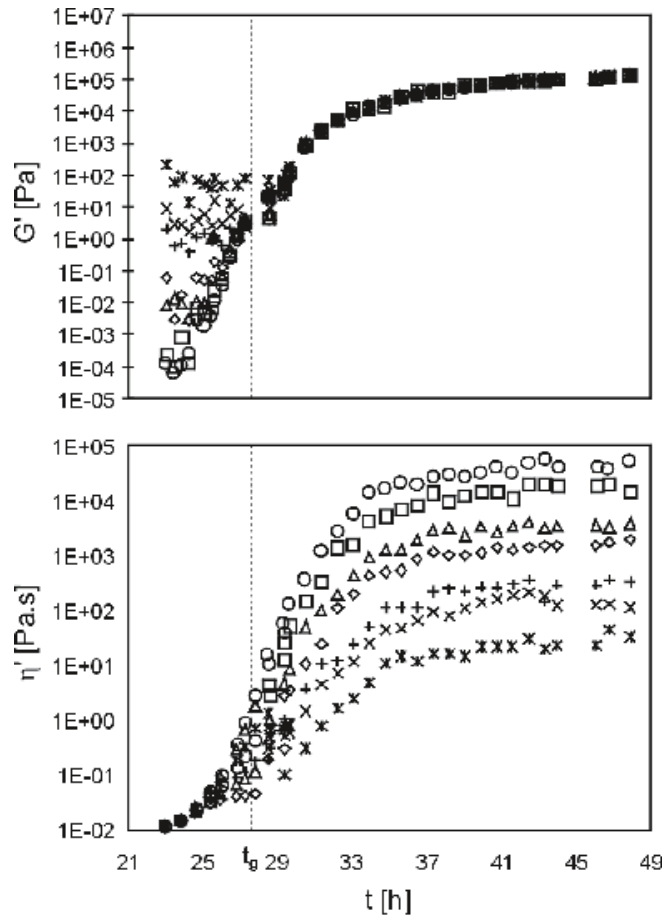

$00.0628 \square 0.135 \Delta 0.52801 .35+6.28 \times 13.5 \times 62.8$

Figura 1. Evolución que siguen las funciones dinámicas: (a) $G^{\prime}(\omega)$ y (b) $\eta^{\prime}(\omega)$ con el tiempo de reacción para los soles de silicio. Los símbolos indican las distintas frecuencias de oscilación ensayadas y la línea discontinua el tiempo de gelificación, $\mathrm{t}_{\mathrm{g}^{\prime}}$ correspondiente.

En particular las Figuras 1 y 2 muestran las evoluciones temporales que siguen el módulo de almacenamiento y la viscosidad dinámica para los soles de silicio y titanio, respectivamente. Al compararlas entre sí, se observa que en el caso de los soles de titanio, dichas propiedades siguen una evolución mucho más suave y gradual que las de los soles de silicio. Por otro lado, en valores absolutos, los soles de silicio alcanzan valores de $G^{\prime}$ y $\eta^{\prime}$ mucho mayores que los de titanio, de forma que los primeros son más viscosos y están constituidos por una red oxídica más reticulada y mecánicamente más resistente.

Las Figuras 3 y 4 muestran las evoluciones temporales que siguen los parámetros viscoelásticos a frecuencia cero, $\eta_{0}$ y $\mathrm{J}_{\mathrm{e}^{0}}^{0}$, para los soles de silicio y de titanio respectivamente. Evidentemente la evolución de $\eta_{0}$ es la misma que la de la viscosidad dinámica, $\eta^{\prime}$ a una determinada frecuencia, ya que ambas miden viscosidad, y la del inverso de $\mathrm{J}_{\mathrm{e}}^{0}$ es como la de $G^{\prime}$ a una determinada frecuencia, dado que ambos miden elasticidad. La única diferencia está en el hecho de que los valores de $\eta_{0} \mathrm{y}\left(\mathrm{J}_{\mathrm{e}}^{0}\right)^{-1}$ están vinculados a la viscosidad y elasticidad de las estructuras más grandes.

Por lo que respecta al módulo plateau, $\mathrm{G}_{\mathrm{N}}^{0}$, se trata de un parámetro viscoelástico que evalúa la elasticidad de la unidad estructural del sistema y corresponde al valor máximo al cual se estabiliza el módulo dinámico elástico a frecuencias elevadas, $G_{N}^{0}=\left|G_{G^{\prime}(\omega)}\right|_{\omega \rightarrow \infty}$. La diferencia que existe entre el módulo plateau y el módulo dinámico es que el módulo dinámico se refiere a la elasticidad de estructuras cada vez mayores a medida que la frecuencia disminuye.

El módulo plateau se obtiene como extrapolación a frecuencias altas de la evolución de $G^{\prime}(\omega)$ frente $\omega$, mediante un
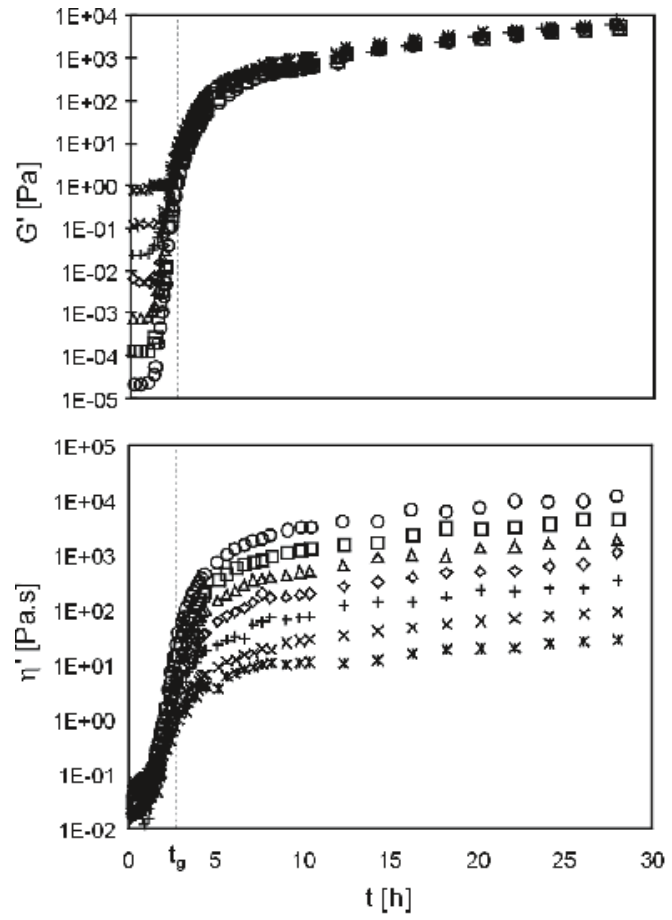

$00.0628 \square 0.135 \Delta 0.292 \bullet 0.628+1.35 \times 2.92 \times 6.28$

Figura 2. Evolución que siguen las funciones dinámicas: (a) $G^{\prime}(\omega)$ y (b) $\eta^{\prime}(\omega)$ con el tiempo de reacción para los soles de titanio. Los símbolos indican las distintas frecuencias de oscilación ensayadas y la línea discontinua el tiempo de gelificación, $\mathrm{t}_{\mathrm{g}}$, correspondiente.

ajuste por mínimos cuadrados, utilizando la función tangente hiperbólica $(6,21)$. Concretamente, la función de ajuste es:

$$
\mathrm{y}=0,5\left(1+\tanh \left(\mathrm{m}_{1}\left(\mathrm{x}+\mathrm{m}_{2}\right)\right)\right)
$$

siendo $y=\left(G^{\prime} / G_{N}^{0}\right)^{0,5} ; x=\log \omega ; m_{1}(>0)$ y $m_{2}$ parámetros de ajuste $\left(\mathrm{m}_{2}\right.$ es el parámetro que sitúa la curva en el eje de abscisas y $\mathrm{m}_{1}$ el parámetro que fija la pendiente en la zona donde $G^{\prime}$ es función de $\omega$ ). Se trata de una función que recoge el comportamiento sigmoidal de $G^{\prime}$ vs. $\omega$, ya que tiene la forma global apropiada y además cumple con los valores límites.

La evolución que sigue $\mathrm{G}_{\mathrm{N}}^{0}$ a lo largo del proceso de gelificación se ilustra en las Figuras 3a y $4 \mathrm{a}$. El aumento progresivo de este parámetro indica que el sistema va reticulando con el tiempo y que la unidad estructural posee una elasticidad cada vez mayor. Comparando los dos tipos de soles estudiados, se deduce que los soles de silicio forman una red oxídica mucho más reticulada y mecánicamente resistente que los de titanio.

\subsection{Obtención de la distribución de tiempos de relajación (DTR)}

El espectro de relajación no puede hallarse de forma directa a partir de medidas reológicas, sino que debe calcularse a partir de alguna de las funciones viscoelásticas del material determinadas experimentalmente $\left(G^{\prime}(\omega), \eta^{\prime}(\omega)\right.$, etc.). No obstante, el cálculo del espectro de relajación no es sencillo, debido a que requiere la inversión de una ecuación integral de Fredholm de primer tipo, como puede observarse a partir de 

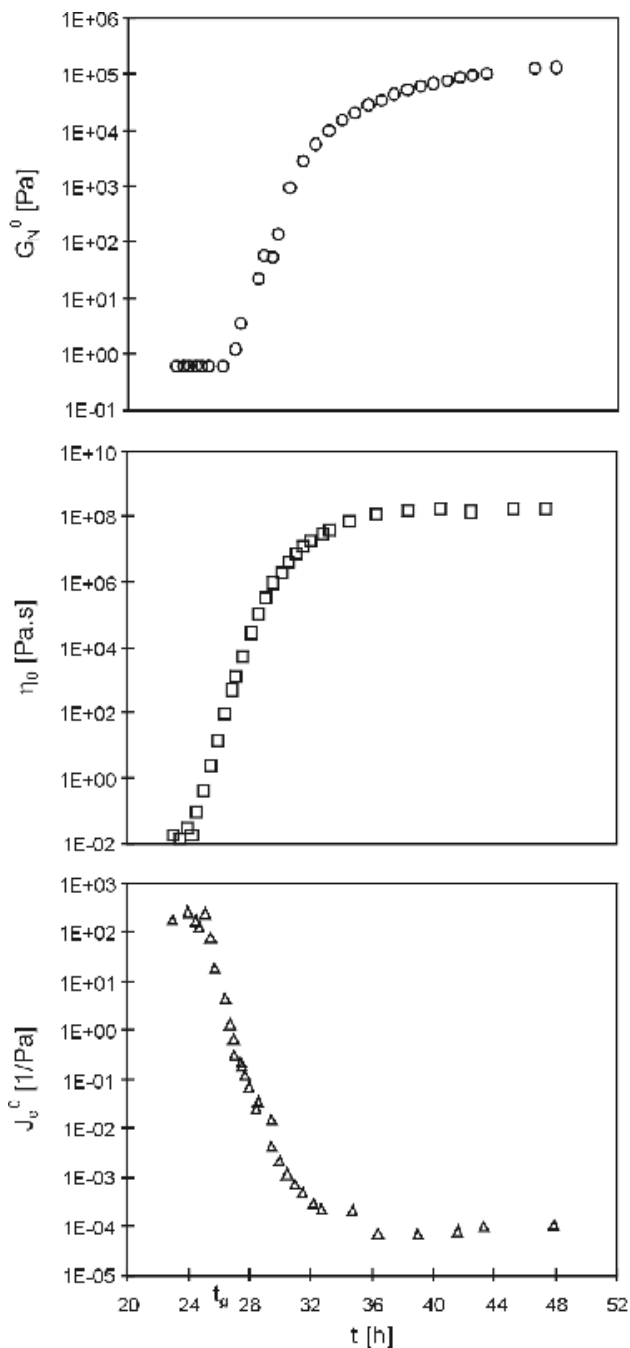

Figura 3. Evolución que siguen los parámetros viscoelásticos con el tiempo de reacción para los soles de silicio: (a) módulo plateau, $\mathrm{G}_{\mathrm{N}^{\prime}}^{0}$ (b) viscosidad límite a gradiente cero, $\eta_{0}$; (c) capacitancia de flujo estacionario, $\mathrm{J}_{\mathrm{e}}^{0}$. La línea discontinua indica el tiempo de gelificación, $\mathrm{t}_{\mathrm{g}^{\prime}}$ correspondiente.

las expresiones que relacionan las funciones viscoelásticas $\eta^{\prime}(\omega)$ y $G^{\prime}(\omega)$ con el espectro de relajación (19):

$$
\begin{aligned}
\eta^{\prime}(\omega) & =\int_{0}^{\lambda_{\max }} H(\lambda) \frac{\lambda}{1, \ldots \jmath^{2}{ }^{2}} \frac{\mathrm{d} \lambda}{\omega^{2} \lambda^{2}} \\
\mathrm{G}^{\prime}(\omega) & =\mathrm{G}_{\mathrm{e}}+\int_{0}^{\lambda_{\max }} H(\lambda) \frac{\mathrm{d} \lambda}{1+\omega^{2} \lambda^{2}} \frac{\lambda}{\lambda}
\end{aligned}
$$

En este trabajo el espectro de relajación se ha calculado teniendo en cuenta simultáneamente las componentes viscosa y elástica del material, de forma que se utiliza la información más adecuada en cada momento, ya que cuando la viscosidad es baja (poca precisión) para una determinada frecuencia $\omega$, la elasticidad es alta (mucha precisión), y viceversa. Así pues, la ecuación integral a resolver es la siguiente:

$$
\mathrm{G}^{\prime}(\omega)+\omega \eta^{\prime}(\omega)=\int_{-\infty}^{+\infty}\left(\frac{\omega^{2} \lambda^{2}+\omega \lambda}{1+\omega^{2} \lambda^{2}}\right) H(\lambda) \mathrm{d} \ln \lambda
$$
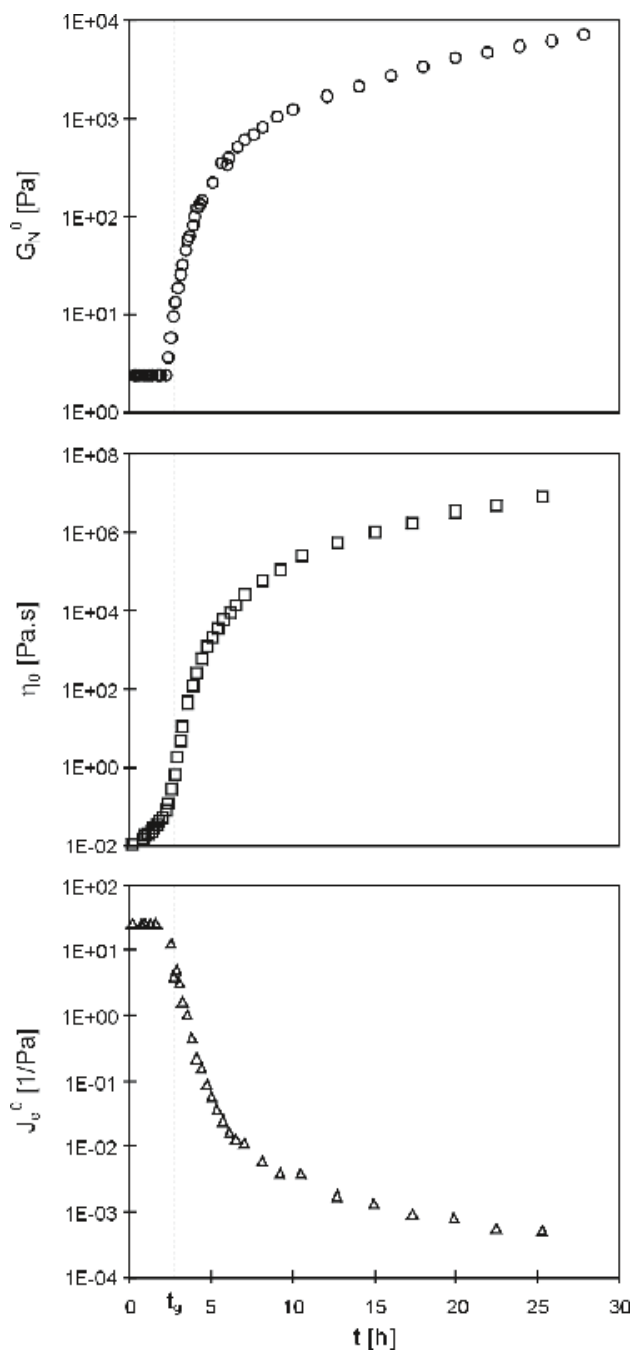

Figura 4. Evolución que siguen los parámetros viscoelásticos con el tiempo de reacción para los soles de titanio: (a) módulo plateau, $\mathrm{G}_{\mathrm{N}}^{0}$; (b) viscosidad límite a gradiente cero, $\eta_{0}$; (c) capacitancia de flujo estacionario, $\mathrm{J}_{\mathrm{e}}^{0}$. La línea discontinua indica el tiempo de gelificación, $\mathrm{t}_{\mathrm{g}}$, correspondiente.

ecuación que resulta de combinar las dos ecuaciones anteriores, donde el módulo de equilibrio, $\mathrm{G}_{\mathrm{e}^{\prime}}$ es nulo para todos los soles estudiados.

La inversión de este tipo de ecuaciones es un problema de naturaleza altamente inestable, debido a que existen muchas soluciones capaces de ajustar correctamente los datos experimentales. Actualmente existen distintos métodos para solucionar este tipo de problema. En este trabajo en particular se ha utilizado el método de la máxima entropía con tiempos logarítmicamente espaciados y obligando a cumplir las diversas condiciones que deben satisfacer las soluciones. Una de ellas es que la solución debe tener la máxima probabilidad dentro del error experimental, propiedad que dirige el problema hacia la solución (22). Esto equivale a buscar la solución que presenta el valor máximo de entropía $\left(-\sum G_{k} \log G_{k}\right)$ entre las muchas soluciones que ajustan los datos experimentales, dentro del error experimental. Además, las otras condiciones que el espectro de relajación debe cumplir son los momentos integrales de la DTR: el módulo plateau, $\mathrm{G}_{\mathrm{N}}^{0}$ (Ec. 10), la viscosidad 
límite a gradiente cero, $\eta_{0}$ (Ec. 11) y el producto capacitancia de flujo estacionario, $\mathrm{J}_{\mathrm{e}}^{0}$, por viscosidad límite a gradiente cero al cuadrado (Ec. 12):

$$
\begin{aligned}
& \mathrm{G}_{\mathrm{N}}^{0}=\int_{-\infty}^{+\infty} \mathrm{H}(\lambda) \mathrm{d} \ln \lambda \\
& \eta_{0}=\int_{-\infty}^{+\infty} \lambda \mathrm{H}(\lambda) \mathrm{d} \ln \lambda \\
& \mathrm{J}_{\mathrm{e}}^{0} \eta_{0}^{2}=\int_{-\infty}^{+\infty} \lambda^{2} \mathrm{H}(\lambda) \mathrm{d} \ln \lambda
\end{aligned}
$$

Para poder evaluar la evolución con el tiempo de los espectros de relajación durante el proceso de gelificación, se han escogido de forma estratégica diferentes tiempos relativos de reacción, $\mathrm{t} / \mathrm{t}_{\mathrm{g}}$, para calcular el correspondiente espectro de relajación, siendo $t_{\mathrm{g}}$ el tiempo de gelificación del sol. Una vez hallado el espectro de relajación, $\mathrm{H}(\lambda)$, en todos los casos se han recalculado a partir de él las funciones dinámicas $G^{\prime}(\omega)$ y $\eta^{\prime}(\omega)$ para comprobar la bondad del espectro hallado. En general se observa una reconstrucción satisfactoria de los datos dinámicos experimentales para cualquiera de los soles estudiados. Por último, para poder comparar los espectros de relajación entre sí, éstos se han normalizado dividiendo por el módulo plateau (ver Ec. 10) correspondiente al tiempo de reacción considerado. En las Figuras 5 y 6 se presenta como evolucionan los espectros de relajación con el tiempo de reacción para los distintos soles de silicio y de titanio estudiados.

El análisis directo de dichos resultados no permite cuantificar las distribuciones de pesos moleculares en valores absolutos. Para ello debería disponerse de un modelo molecular para sistemas ramificados que relacionase tiempos de relajación con pesos moleculares y distribuciones de tiempos de relajación con distribuciones de pesos moleculares, y hasta el momento actual, no se ha desarrollado ningún modelo para este tipo de sistemas, dada su gran complejidad. De hecho, toda la investigación que se está realizando actualmente en este campo está siendo dirigida hacia sistemas lineales.

Ahora bien, el análisis de la distribución de tiempos de relajación permite obtener información sobre el tamaño relativo de las estructuras, el tipo de distribuciones (más o menos polidispersas), la cantidad de materia presente en el sistema con un determinado tiempo de relajación, así como poder evaluar la influencia que ejercen las condiciones de gelificación.

Al analizar la evolución de los espectros de relajación durante el proceso de gelificación, se observa que la forma cualitativa como evolucionan los espectros para los distintos sistemas estudiados es independiente de la naturaleza del sol, y que describe perfectamente la pauta general de evolución estructural que sigue un sistema que está gelificando. Así pues, se observa que al principio, en los estadios pre-gel, el sistema es bastante monodisperso (distribuciones considerablemente estrechas), predominando las estructuras pequeñas con tiempos de relajación pequeños. A medida que el sistema gelifica, se forman estructuras de peso molecular cada vez mayor (con tiempos de relajación mayores), en detrimento de las estructuras con tiempos de relajación pequeños, ya que se observa una disminución de $\mathrm{H}(\lambda)$ para valores pequeños de $\lambda$ o incluso su total desaparición. Para tiempos de reacción elevados (estadios post-gel), las estructuras que predominan en el sistema son las que poseen tiempos de relajación mayores y, por tanto, de mayor peso molecular, situación inversa a la observada para los tiempos iniciales de reacción. Por tanto, lo que se observa es un progresivo desplazamiento horizontal de las distribuciones de tiempos de relajación con el tiempo de reacción hacia tiempos de relajación mayores, tal como era de esperar. (a)

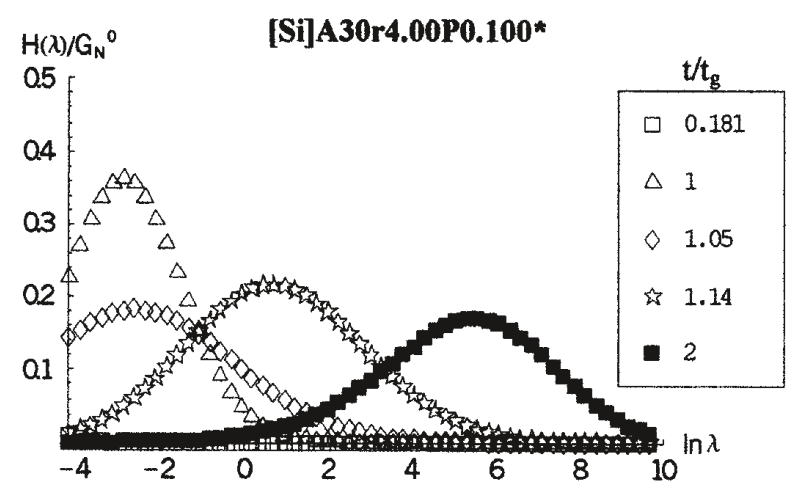

(b)

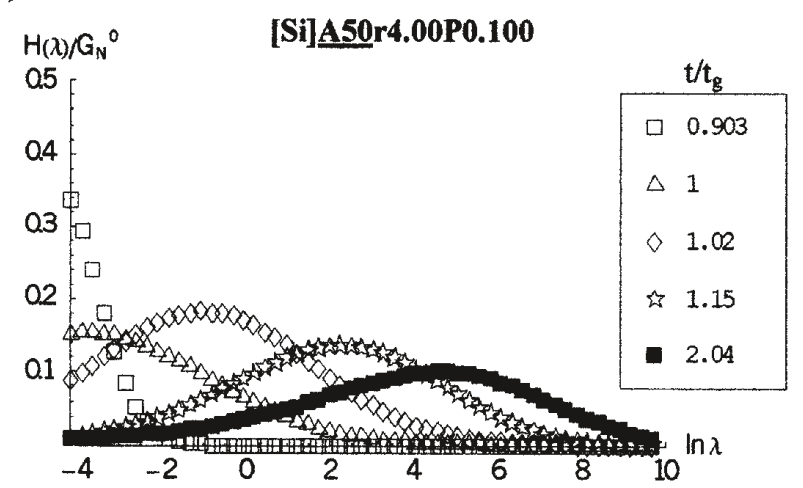

(c)

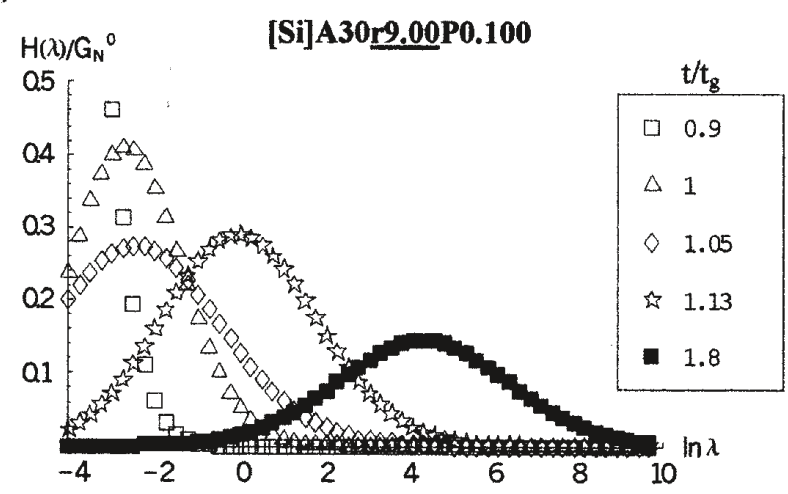

(d)

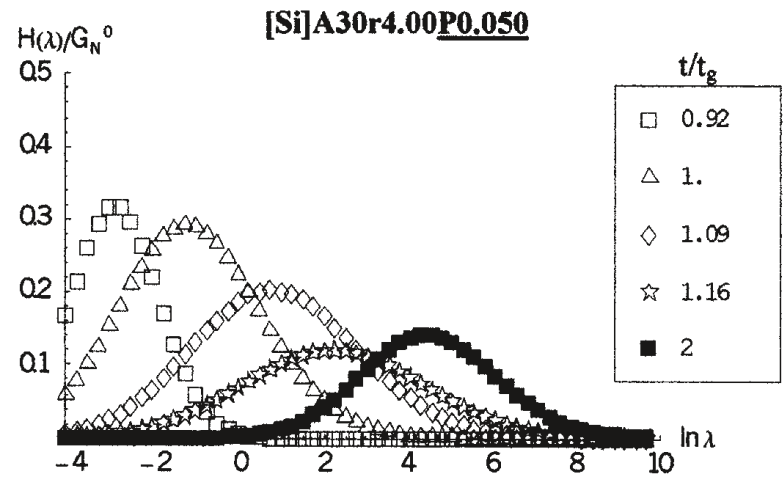

Figura 5. Evolución con el tiempo de reacción adimensional, $\mathrm{t} / \mathrm{t}_{\mathrm{g}^{\prime}}$ de los espectros de relajación para los distintos sistemas poliméricos de silicio estudiados. 
(a)

(b)
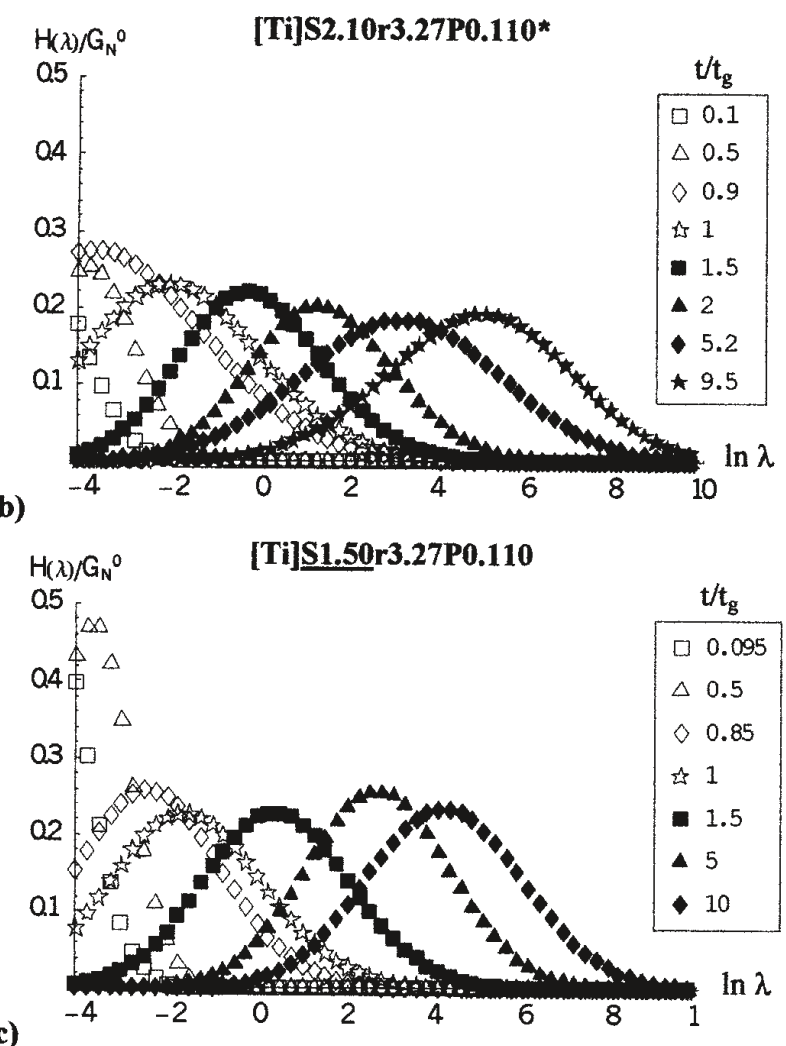

(c)

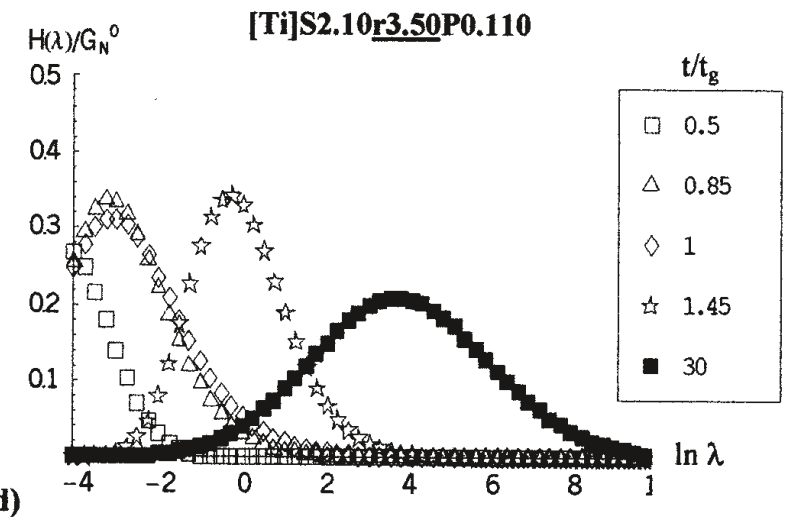

(d)

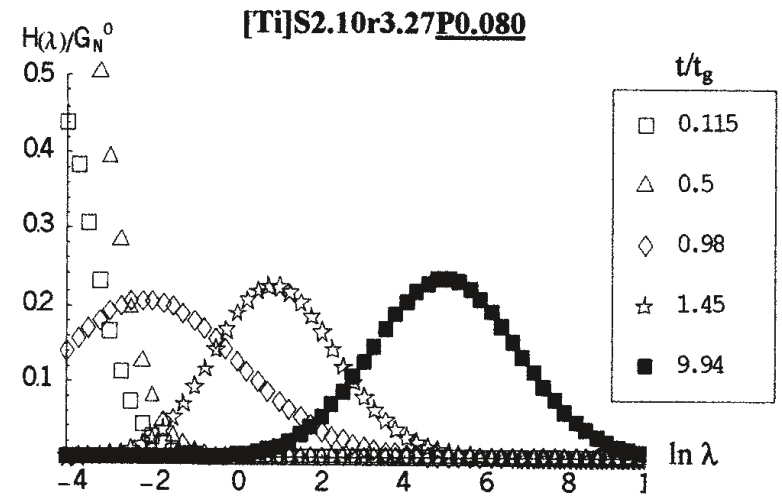

Figura 6. Evolución con el tiempo de reacción adimensional, $\mathrm{t} / \mathrm{t}_{\mathrm{g}^{\prime}}$ de los espectros de relajación para los distintos sistemas poliméricos de titanio estudiados.
Es importante destacar que para tiempos próximos al punto de gelificación se observa un ensanchamiento significativo de las curvas, hallándose que el tiempo de gelificación es precisamente el tiempo de reacción que corresponde a un ensanchamiento máximo de la distribución de tiempos de relajación, donde todas las estructuras moleculares están presentes de forma significativa. Este rasgo diferencial es muy interesante ya que permite una nueva definición de tiempo de gelificación basada en la evolución de los espectros de relajación.

A continuación se analiza la influencia que ejerce la composición del sol sobre la evolución de los espectros de relajación. En primer lugar cabe decir que la composición del sol tiene una fuerte influencia sobre el tiempo de gelificación, tal como se puede apreciar en las Tablas III y IV, donde se tabulan los tiempos de gelificación de los soles de silicio y titanio respectivamente. Por este motivo se han calculado los espectros de relajación para tiempos relativos de reacción $\left(t / t_{\alpha}\right)$ correspondientes a estadios concretos del proceso de gelificación, independientemente de la cinética que siga la gelificación, y de esta forma poderlos comparar entre sí.

Una distribución de pesos moleculares queda caracterizada con dos parámetros: un peso molecular medio (cociente entre dos momentos consecutivos de la distribución) que sitúa la distribución a un nivel de pesos moleculares y la polidispersidad (cociente entre dos pesos moleculares medios) que establece la amplitud de la distribución. Análogamente, la distribución de tiempos de relajación queda caracterizada con un tiempo de relajación medio y su polidispersidad. Como tiempo de relajación medio se tomará el tiempo de relajación correspondiente al máximo de la distribución, el cual dará una idea sobre el tamaño medio de las estructuras. Para analizar la influencia de las condiciones de gelificación sobre estos parámetros característicos de la distribución se comparará cada uno de los soles estudiados con el sol estándar.

En cuanto a los soles de silicio (ver Figura 5), se observa que un aumento de la proporción de alcohol presente en el sistema proporciona un aumento considerable en la polidispersidad a cualquier tiempo relativo de reacción, así como tiempos de relajación medios mayores. Por tanto, para un mismo tiempo de reacción, el sol con mayor proporción de alcohol contiene estructuras de un tamaño medio mayor, coexistiendo estructuras más pequeñas y más grandes que en el sol estándar. Un aumento en la proporción de agua comporta distribuciones menos polidispersas y tiempos de relajación medios menores, siendo pues las estructuras de menor tamaño que las presentes en el sol estándar. Por último, el efecto del catalizador sobre la polidispersidad de las distribuciones es menor. Se observa que en este caso las polidispersidades son ligeramente menores que las correspondientes al sol estándar. En cuanto a los tiempos de relajación medios, éstos son mayores, excepto para el tiempo adimensional de reacción 2, que es ligeramente menor. Así pues, en general, las estructuras son de un tamaño medio mayor.

En los soles de titanio (ver Figura 6) se observa que cualquiera de los cambios realizados en la composición del sol (menor cantidad de modificador del alcóxido, mayor proporción de agua y menor proporción de catalizador) conlleva distribuciones más monodispersas. Por lo que respecta a los tiempos de relajación medios, no se observa una tendencia clara. Al disminuir la cantidad de modificador presente en el sol, el tiempo medio de relajación es ligeramente superior para los estadios pre-gel y post-gel cercanos a la gelificación, mientras que para tiempos adimensionales de reacción superiores a 1,5 el tamaño medio de las estructuras 
es claramente inferior. Un aumento en la proporción de agua supone un menor tamaño medio de las estructuras que conforman el sistema. Por último, al igual que ocurre para los soles de silicio, la influencia que ejerce la cantidad de catalizador presente en el sol es mucho menor que la del resto de variables. De hecho, no se observa ninguna diferencia apreciable con el sol estándar para los estadios pre-gel y gel, siendo el tamaño medio de las estructuras muy parecido. Sólo para el estadio post-gel $\left(t / t_{g}=10\right)$ se observa un tamaño medio de estructuras algo menor.

\section{CONCLUSIONES}

La técnica propuesta en este trabajo para el seguimiento continuo del proceso de gelificación mediante la evolución de los espectros de relajación permite seguir la evolución con el tiempo de las estructuras que se van formando. Dicha técnica abre muchas posibilidades en un nuevo campo que podría denominarse reocinética.

Las funciones y propiedades viscoelásticas de todos los sistemas estudiados, independientemente de la naturaleza y composición del sol, siguen la pauta general en la que, al principio, la viscosidad y la elasticidad del sistema son muy bajas y prácticamente constantes, aumentando de forma brusca en el punto de gelificación, momento a partir del cual continúan aumentando pero ya de forma mucho más suave. También la evolución de los espectros de relajación describe la misma pauta: inicialmente el sistema es bastante monodisperso, formándose estructuras de peso molecular cada vez mayor a medida que el sistema reacciona.

Se propone una nueva visión descriptiva del tiempo de gelificación, basada en la evolución de los espectros de relajación, que consiste en caracterizar el punto de gelificación como el tiempo de reacción en que la distribución de tiempos de relajación presenta una polidispersidad máxima.

Al comparar los dos tipos de soles estudiados, se concluye que la cinética del proceso de gelificación de los soles de silicio es mucho más lenta que la de los soles de titanio, siendo, sin embargo, la transición mucho más brusca. Por otro lado, a partir de la evolución que siguen las funciones y propiedades viscoelásticas de los soles, se concluye que los geles de silicio son más viscosos y elásticos, duros y resistentes que los de titanio, dando lugar a geles con un mayor densidad de reticulación.

En cuanto a la influencia que ejerce la composición del sol sobre la polidispersidad y el tiempo de relajación medio, parámetros que caracterizan la distribución de tiempos de relajación, se concluye que se consiguen distribuciones de estructuras moleculares más monodispersas al aumentar la razón molar de agua o bien al disminuir la razón molar de catalizador, independientemente de si se trata de soles en base silicio o en base titanio. En cuanto a los parámetros específicos de los dos tipos de soles estudiados, porcentaje de alcohol, A (soles de silicio) y razón molar de modificador del alcóxido, $\mathrm{S}$ (soles de titanio), se concluye que en ambos casos una reducción del parámetro proporciona distribuciones más monodispersas. La uniformidad observada en el efecto de las condiciones de gelificación sobre la polidispersidad del sistema no se presenta en el efecto que ejercen sobre el tiempo de relajación medio y, por tanto, sobre el tamaño medio de las estructuras que conforman el sistema. De todos modos, se observa en los sistemas de silicio que un sol con mayor proporción de alcohol presenta un tamaño medio mayor. El mismo efecto se observa al disminuir la razón molar de catalizador. Por el contrario, se obtienen geles con un tamaño medio de las estructuras menor al aumentar la razón molar de agua, r. Por lo que respecta a los sistemas de titanio, se concluye que los cambios efectuados en la composición de los soles no comportan diferencias significativas en cuanto al tamaño medio de las estructuras.

\section{BIBLIOGRAFÍA}

1. J.P. Montfort, G. Marin, P.Monge, “Molecular weight distribution dependence of the viscoelastic properties of linear polymers. The coupling of reptation and tube renewal effects", Macromolecules, 19, 1979-1988 (1986)

2. R.H. Colby, L.J. Fetters, W.W. Graessley, "Melt viscosity-Molecular weight relationship for linear polymers", Macromolecules, 20, 2226-2237 (1987)

3. D.W. Mead, "Determination of molecular weight distributions of linear flexible polymers from linear viscoelastic material functions", J. Rheol., 38[6], 1797-1827 (1994)

4. S.T. Milner, "Relating the shear-thinning curve to the molecular weight distribution in linear polymer melts", J. Rheol., 40 [2], 303-315 (1996)

5. P.M. Wood-Adams, J.M. Dealy, "Use of rheological measurements to estimate the molecular weight distribution of linear polyethylene", J. Rheol., 40 [5], 761-778 (1996)

6. C. Lavallée, A. Berker, "More on the prediction of molecular weight distributions of linear polymers from their rheology", J. Rheol., 41 [4], 851-871 (1997)

7. C. Carrot, J. Guillet, "From dynamic moduli to molecular weight distribution: A study of various polydisperse linear polymers", J. Rheol., 41 [5], 1203-1220 (1998)

8. J.E. Martin, D. Adolf \& J.P. Wilcoxon, “Viscoelasticity near the sol-gel transition", Phys. Rev. A, 39 [3], 1325-1332 (1989)

9. W. Hess, T.A. Vilgis \& H.H. Winter, "Dynamical critical behavior during chemical gelation and vulcanization", Macromolecules, 21, 2536-2542 (1988)

10. D. Adolf, J.E. Martin \& J.P. Wilcoxon, "Evolution of structure and viscoelasticity in an epoxy near the sol-gel-transition", Macromolecules, 23, 527-531 (1990)

11. C. Guizard, J.C. Achddou, A. Larbot \& L. Cot, “Sol-to-gel transition in reversed micelle microemulsions. III. Rheology", J. Non-Cryst. Solids, 147/148, 681-690 (1992)

12. A. Bleuzen, S. Barboux-Doeuff, P. Flaud \& C. Sanchez, "Rheological study of titanium oxide based gels", Mater. Res. Bull., 29 [12], 1223-1232 (1994)

13. M. Jokinen, E. Gyorvary \& J.B. Rosenholm, "Viscoelastic characterization of 3 different sol-gel derived silica-gels", Colloids and Surfaces A: Physicochemical and Engineering Aspects, 141 [2], 205-216 (1998)

14. J. Llorens, "Molecular weight distributions from viscoelastic parameters in polymeric sols as the reaction proceeds", J. Non-Cryst. Solids, 162, 188-196 (1993)

15. D.W. Mead, "Component predictions and the relaxation spectrum of the double reptation mixing rule for polydisperse linear flexible polymers", J. Rheol., 40 [4], 633-661 (1996)

16. J.C. Ro \& I.J. Chung, "The rheology of silica sols during the gelation process", J. Non-Cryst. Solids, 126, 259-266 (1990)

17. T. Kamiyama, M. Mikami \& K. Suzuki, "A SAXS study of the gelation process of silicon and titanium alkoxides", J.Non-Cryst. Solids, 150, 157-162 (1992)

18. S.Y. Chang \& T.A. Ring, "Map of gel times for three phase region tetraethoxysilane, ethanol and water", J.Non-Cryst. Solids, 147\&148, 56-61 (1992)

19. J.D. Ferry, Viscoelastic properties of polymers, $3^{\mathrm{a}}$ edición, John Wiley \& Sons, New York (1980)

20. E. Rudé, J. Llorens \& C. Mans, "Viscoelastic properties in the course of hydrolysis and condensation reactions of modified titanium alkoxides leading to gelation", Colloids and Surfaces A: Physicochemical and Engineering Aspects, 119, 57-65 (1996)

21. W.H.Tuminello, "Molecular weight distributions of tetrafluoroethylenehexafluoropropylene copolymers", Polym. Eng. Sci., 29[10], 645-653 (1989)

22. J. Skilling, R.K. Bryan, "Maximum entropy image reconstruction: general algorithm", Mon. Not. R. Astr. Soc., 211, 111-124 (1984).

Recibido: 02.08 .99

Aceptado: 06.04 .00 